\title{
Two pseudo-students: Applications of machine learning to formative evaluation
}

\author{
Kurt VanLehn \\ Departments of Psychology and Computer Science \\ Carnegie-Mellon University \\ Pittsburgh, PA 15213 U.S.A.
}

\section{The need for on-line evaluation of instructional designs}

Ideally, the design of instructional material should include ample testing of preliminary designs so that design errors can be detected and repaired before the instruction is deployed. Such formative evaluations are rarer than they should be for several reasons:

- Although the final polishing can be left off, all the little design details must be fully worked out before the instruction can be given to human students. It would be much better if formative evaluations could be conducted while the design was still in a rough, preliminary state. When design errors are detected early in the design cycle, less time is wasted working out the details of designs that will ultimately be discarded.

- Testing instruction with human students can be difficult, time consuming and expensive. Although increasing the quality of instruction is always a desirable goal in principle, the time and money needed for formative evaluation may not always be justifiable.

- Current testing methods often yield only two kinds of information, neither of which is particularly helpful to designers. First, there is a overall assessment of instructional effectiveness, such as a difference between pre-test and post-test scores. Second, there are the participants' comments as to which parts of the instruction seem to work well, and which did not.

In contrast to the situation in instructional design, consider the state of the art in mechanical and electrical design. After drafting blueprint or schematic of their design on a CAD station, designers can submit it to a simulation program for evaluation. Simulation programs provide very detailed reports on the design's performance which allow the engineer to locate design errors early in the design process. They may not find all the errors, because the simulation programs and testing regimes are not always perfect, but catching errors early is much cheaper than catching them later or not catching them at all. 
for formative evaluation during the instructional design process. Such simulations are called pre'tule-students, beciase they simulate human students learning from the given instruction. llowever, unlike human students, pseudo-students ketp a detailed trace of the learning so that the designer can discover the causes of undesirable pedagogical outcomes. For instance, one pseudo-student, Sierra, helped demonstrate that many systematic arithmetic errors are ciaused by incomplete and poorly setyuenced instruction (VanLehn, 1990a). Most of these design defects would be easy to fix now that they have been detected.

\section{Types of pseudu-students}

Just as there must be multiple simulation programs in mechanical and electrical engineering, there slivuld be multiple psetudo-student programs, eitch adapted for a particular class of instructional design problems. An instructional design problem is framed by two impontant choices: the delivery mode and the subject matter. There are many different options for both. For instance, instruction can be delivered by lectures, textbooks, sinulation-based trainers, printed tutorials, human tutors or peer teaching groups. Subject matter can range from text editor commands to algebraic problem solving to mechanical design. It is not practical to develop pseudo-students that can be applied to all problems in this vast two-dimensional spitc. llowever, it is feasible to address well-defined regions characterized by particular classes of delivery modes and subject matters. This paper discusses two pseudo-students, Sicrra and Cascade. The subject mater of Sierra is arithmetic algorithms, and the delivery mode is standard textbook-based classroom instruction. The subject matter of Cascade is college physics problem solving, and the delivery mode is self-study of the textbook.

There are many individual difierences among students, and it would be pointless for a pseudo-student to model just one kind of student. Thus, a proper pseudo-student must have paramelers for representing individual diflerences among students. A thorough evaluation of a proposed piece of instruction reyuires gathering the reactions of a whole "school" of pseuda-students, whete differen members of the sithool have different parameter setings. However, if there is too much variability among the students, it may be infeasible to capture it all with mere parameler variations. Structurally different pseudo-students may be necessitry. In physics problem solving, there are two rather distinct learning learning styles (Chi, Bassok, Lewis, Reimamn \& Glaser, 195\%), so the current version of Cascade has two difierent learning models. Although less is known about arithmetic learning styles, it appears that there ate itso two approithes, a "syntactic" une and a "semantic" one (Resnick, 1982). The semantic or meaningful approach gentrally leads to acquiring correct knowtedge of the algorihms. The syntactic approath sometimes leads to correct knowledge, but it often leads to acyuiring incorrect, "bugagy" algyorithms. Sicrat models only the syntactic approach.

Given all the recent work in mathine learning, it is certainly technologically feasible to build programs that learn from the same kind of instruction that human subjects do. Moreover, it would be a simple matler to ydd a trate to their processes so that one could find the causes for the learning oucomes producid while running the program. In fact, it would not even be necessary to have the pseudo-student produce meisurable outcomes, such as test scores, in order to tell if it had learned correctly. One can simply inspect the knowledge structures it produces instead. If the acquired knowledge is incorrect, one can inspect the traces, find the causes of the incorrect knowledge, and suggest changes in the instructional design.

No one would trust such suggestions unless the pseudo-student had been very carefully validated with human students. Even if its learning processes were be consistent with what is known about human psychology, one would still need assurances that its predictions about learning outcomes are in fact accurate predictions of the learning outcomes from human students. In short, an important phase in the development of a pseudo-student is comparing its learning outcomes to human students.

This introduces another important dimension along which pseudo-students can differ. They can produce different learning outcomes for comparison with human students. They might predict achievement test results, transfer, the time require to learn to criterion, error profiles, or any other outcome that is both measurable in both human and machine behavior. For instance, Sierra predicts systematic errors, and Cascade predicts problem solving protocols.

In summary, there are four dimensions along which pseudo-students differ: (1) the subject matter area, (2) the delivery mode, (3) the class of learning styles or approaches, and (4) the outcome measure used for assessing the accuracy of the pseudo-student as a model of human students.

\section{Sierra}

There are two big problems in teaching arithmetic procedures. The problem that has received the most research is how to get students to acquire a meaningful understanding of the procedures. However, a more basic problem is that some students do not learn the correct procedure at all. Instead they acquire a buggy procedure, which may resist detection and remediation for years (Brown \& Burton, 1978; VanLehn, 1982). Buggy procedures cause errors that are systematic: the student will produce the same incorrect answer when given the same problem twice. Sometimes the class of problems that cause incorrect answers is very small relative to the whole class of arithmetic problems, so detecting a buggy procedure can be difficult. Worse still, there is evidence that bugs often spontaneously resurface even after apparently successful remediation (Resnick \& Omanson, 1987). These difliculties in detecting and remediating bugs have led researchers to suggest that instruction be redesigned so that bugs never have the opportunity to be learned (Resnick \& Omanson, 1987). This is the design problem that motivates Sierra.

Sierra was originally intended as psychological model of the learning processes that cause bugs and as an exercise in a new method of psychological investigation called competitive argumentation (VanLehn, Brown \& Greeno, 1984). Now that it has been built, its more practical benefits have become apparent. This paper explores its potential as a pseudostudent. 
There are several assumptions about kearning that are deeply ingrained in Sierra. The most important one is that only the hinds of learning that can lead to bugs are modeled. In particular, Sierra does not include anything like the kinds of meaningful procedural learning that are ascribed to spme goud studems. That kind of kearning presumably produces only contect procedures. If we watht to detect instructional design errors that can cause errors, it does no good to include a kind of learning that is not sensitive to those errors. We want to design instruction so that even students who camnot or will not learn meaningfully can at least get a correct procedure out of the instruction.

A second assumption is that students who acquire bugs are driven mosily by the examples and exercises given to them, and they make liuke use of the natural language explanations that textbooks and teachers offer them. This assumption is consistent with many laboratory and rield studies (see VanLehn, 1986, for a review).

In order to actually use an mathematical procedurc, ont must know more than the procedure itself. One must also understand the symtax of the mathematical notation. For instance, the row's and columns are important in subtraction, but diagonals are not. For many kinds of mathematics, one must also know the addition, subuaction and multiplicution tables (e.g., that $3+5=8$ ). Sierra models only the process of learning procedures, so it is assumed that prereyuisite knowledge of notation and number facts has been mastered.

These assumptions imply that Sierra's learning task is to induce a procedure from examples of its operation. This learning task has been widely studied in the machine learning literature (sce VanLehn, 1987, for a review), and there are two basic methods for solving it. One is explanation-based learning (EBL). EBL begins by explaining the first of the given examples. Is explanation is based on a complete understinding (called a domain theory) of the subject matter. The explanation is generalized slightly and stored in memory. The next time it encounters a problem that is similar to the example's problem, it can retrieve the generalized example and re-use it solution. This is more efficient than deriving the solution from first principles. Thus, EBL improves the overall efficiency of the problem solver, allowing it to solve problems that might otherwise be beyond its reach.

The sccond main learning techuigue in machine learning is called similarity-based learning (SBL). SBL compares ex:mples to each other, notes their similarities and differences, and forms aencralizations.

Neiblur EBL nor SBL are exactly rigla for modcling how humans learn mathematical procidurs. The problem is that most procedures are taught incrementally. First a simple version of the procedure is taught. For instance, the first version of a suberaction procedure might only hande problems that do me reguire borrowing (e.g., the simple procedure can

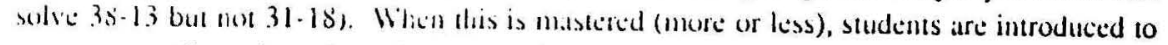
a more complicated version of the procedure, such as one that can handle simple cases of borruwily (e.g., 31-18) but not mure complicated cilses (e.g., 301-8). This incremental keiching process of continuts untit the complete procedure has been presented.
The incremental nature of procedural teaching makes SBL inappropriate because SBL has no way to utilize material that it learned earlier. When an SBL algorithm is trying to master the second lesson, it has no way to use the procedures that it acquired during the first lesson. Clearly, this is not how students learn procedures.

On the other hand, EBL is also inappropriate. For procedural EBL, the domain theory is a procedure. EBL creates specialized versions of the procedure adapted for particular types of problenıs, which may make problem solving more efficient for those problems. However, it cannot actually learn the procedure itself. Yet that is exactly what mathematics students are doing, so EBL is not an appropriate model of their learning.

In order to build Sierra, a new machine learning technique was invented (actually, it was invented concurrently by several investigators -- see VanLehn, 1990a, for discussion). It combines EBL and SBL. The basic idea is to explain an example as much as possible using the current procedure. If the explanation cannot be completed, which will happen whenever the lesson is introducing a new version of the procedure, then the algorithm finds the smallest gap in the explanation such that if that gap were filled, the explanation would be complete. It collects such gaps from several examples, then it uses SBL to assess their similarities and differences, and make a generalization. The generalization is procedural, but it is not a whole procedure for solving the problem; rather it is solves only the little bit of the problem spanned by the gaps. So this piece of procedure is called a subprocedure. The subprocedure generated by SBL is added to the old procedure, creating a new procedure that is be able to explain all the lesson's examples because the new subprocedure suffices to fill all the gaps left in the old explanations. This technique is called explanation completion (EC).

In order to use Sierra, one provides two formal encodings. The first represents of the prerequisite notational and factual knowledge knowledge. The second represents the instruction. Since Sierra ignores natural language, the instruction consists of a sequence of lessons, where each lesson contains only examples and exercises. An example is a sequence of problem states. The first problem state is the initial problem to be solved, and the remaining states show the effects of consecutive writing actions. For instance, here is an example of a subtraction problem being solved:

\begin{tabular}{rrrr} 
& 2 & 2 & 2 \\
$3^{1} 4$ & $\gamma^{1} 4$ & $x^{1} 4$ & $x^{1} 4$ \\
-17 & -17 & -17 & -17 \\
\hline
\end{tabular}

Exercises are just unsolved problems, such as the initial state above.

Sierra represents individual differences among students by being non-deterministic. In the learning algorithm, there are several choice points. For instance, there might be more than one way 10 generalize the set of gaps. Some students may pick one generalization, and other students might pick a different generalization. When Sierra encounters such a choice point, it makes a copy of itself on another computer. One copy takes one of the choices, and the other copy takes the other choice. Although Sicra runs best if it has many computers 
dedicated to it, it can run on a single compuler by saving copies of itself on disk and running them later.

In order to measure the accuracy of Sierra as a model of human students, its learning was compared that of 1,147 young students (ages 6 to 9) who were learning subtraction from two standard American textbooks. A cross-sectional design was used. Students at various stages in their training were all given the same diagnostic test. Their answers were analyzed by Debuggy (Burton, 1982) and a bug profile was developed for each student. Meanwhile, the examples and excrises were extracted from the texibooks and formalized. These two instructional designs wete given to Sierra, which learned 63 distinct procedures from it. These procedures "took" the same diagnostic test as the students, the answers were analyzed by Debuggy, and bug profiles were developed. Depending on how exactly how one compares the two sets of bug protiles, Sierra modeled between $52 \%$ and $85 \%$ of the student's bugs (VanLehn, 19901). Although there is clearly room for improvement, this degree of accuracy is much beller than Sierra's predecessors and probably good enough that it could be used as a tool for formative evaluation. Alter all, mechanical and electrical simulators are not perfect models, yet they are still useful to designers nonetheless.

It Sierra hatd been used as a design tool, whiat would it have suggested to the authors of the two suberaction lesson scyltences? lis major suggestion would be to avoid premature problem solving. Many of the bugs were caused, according to Sierra, by having students solve problems before they had mastered the version of the procedure needed for solving them. These bugs could have been prevented by, for instance, giving more examples or having a tutor supervise the practice of the initial exercises. In particular, many bugs were generated by testing itself. On many tests, including our diagnostic test, students are asked to solve problems that are beyond their level of training. This causes them to reach impasses, which they surmount by inventing simple subprocedures that are usually incorrect (Brown $\&$ VanLehn, 1980). The result is that they learn a wrong way to handle these problems, which in turns may make it more difficult to learn the right way later.

Sierra made several more local suggestions. For instance, both textbooks introduce the borrowing subprocedure ower a multi-lesson sequence that gradually increases the complexity of the problems. This caused many bugs. Sierra would build an overly specific procedure during the first lesson, then have troubles gentralizing it on later lessons. It would be betler to introjuce the subprovedure in a lesson that contains both simple and complex problems. This would allow Sicrat to induce the right level of generality during the initial lessun.

Although Sierra would in principle be a useful tool for instnctional designers, Sierra was exploratory sofitware, pushing the limits of machine learning and arificial intelligence. It wats slow, unfriendly, and written in a now-obsclete computer language (Interlisp-D). I could perhaps be developed into usable sofiware, but it would take a significant effor. More imporanuly, much has been learned about human learning since Sierra was completed in 1985. Some of those results should be incorporated into a revised version.

\section{Cascade}

The primary goal of the Cascade project is to understand the cognitive processes of students who are learning physics problem solving by studying examples and solving exercises. One of the products of this project will be a simulation of the learning processes. Eventually this simulation could become a physics pseudo-student.

Several recent studies have shown that a major difference between good learners and poor learners is how they study the examples. Chi, Bassok, Lewis, Reiman and Glaser (1989) were the first to discover this difference. They took protocols of 8 students as they read a chapter on translational dynamics in a standard college textbook, studied 3 examples, and worked 19 problems. Subjects were categorized as either Good or Poor students on the basis of a median split on their scores while solving the problens. Since students were not significantly different on pre-tests, we can infer that the Good students were better at leaming than the Poor students. Chi et al. analyzed the protocols to see if the Good students were acting differently from the Poor students as they studied. Two significant differences were found. First, the Good students tended to explain the examples very thoroughly to themselves. They would check each line of the example's solution to see if it could be derived from the preceding lines. The Poor students, on the other hand, tended to simply paraphrase the line, then move on to the next one. Often, they just read the line without even paraphrasing it. There was a second significant difference found by Chi et al. When students commented about their understanding of the line they were studying, the Good students would tend to say that they did not understand the line, while the Poor students tend to say that they did understand it. These two findings have been replicated in two other domains: Lisp (Pirolli \& Bielaczyc, 1989) and electrodynamics (Ferguson-Hessler \& de Jong, 1990). The two findings are consistent with the hypothesis that a major difference between Good and Poor students is that only the Good students explain the examples to themseives, checking their knowledge by seeing if it is complete enough to regenerate the example's solution. Chi et al. call this strategy self-explanation.

The main goal of the Cascade project is to model the learning processes of the Good and Poor subjects. Chi et al. graciously gave us copies of their protocols, which are being used to evaluate the accuracy of the models. The project is in progress, so only a preliminary repon can be given.

The model of the Poor students is quite simple. As they read the examples, they retain only those lines that have equations. These are added to the stock of eyutitions obtained by reading the chapter's prose. When the Poor student model solves examples, it selects an equation that contains the sought quantity, then recurses to find values for the other quantities in the equation. This means-ends style of problem solving is quite common and has been modeled before (Larkin, 1986; Bundy et al., 1979).

We have tried three models of self-explanation so far. One was a simple form of explanation-based learning (van Harmelen \& Bundy, 1988). It required such a complete version of the domain knowledge that it now seems quite implausible. For instance, we had 
to assume that the student alleady knew about centain forces laws (i.e., that a surface exerts on an object a force that is perpendicular to the surface) before they staned studying the examples even though the actual text first introduces these laws in the midst of the examples. Of the 111 rules in the knowledge base, 17 were introduced for the first time in the examples. Most of these rules were simply used without comment. Since EBL merely operationalizes knowledge that it already possesses, it could not explain how students learn these 17 rules.

The next model wats based on explanation completion (EC), although of a much simpler kind than the EC algorithm used in Sicra. When a gap is encountered, the EC algorithm selects a tcmplate or "explanation pallern" (Schank, 1986), fills in its slots so that it will bridge the rap, and saves this structure as a new nule. This model sufficed to learn 4 of the 17 rules. The current model is a combination of EC and LEBL, called explanation-based learning of correctness (EBLC). It suffices to learn all 17 rules (Vanlethn, 1990b).

The next step in the research will be to find a way to combine the Good student model and the Poor student model. This is not as easy as it may scem. The Good student model "thinks" like a physicist, in terms of furces and accelerations. The Poor student model "thinks" like a novice, in terms of variables and equations (Larkin, 1986). Although we are fairly sure that this is an alceurate characterization of the 8 human subjects towards the end of the experiment, we are quite uncertain about. where it developed. We doubt that the students came to the experiment with a preset algebraic or physical approaches to the material since their scores on the pretests are not significantly different. However, we do not see yet how self-explanation can cause one group to develop a physics approach while the lack of selfexplanation causes the other group to develop an algebraic approach.

It night seem that the major news for education is simply that physics students should be encouraged (or coerced) into explaining examples to themselves. Perhaps all the detail one gets from a pseudo-student analy'sis is not useful for this instructional design problem. On the other hand, it could be that getting students to sclf-explain is only the beginning. Selfexplanation is an improvemen over paraphrasing ex:mples, but there will probably be ways to improve it. For instance, it is clear alteidy from the Cascade simulations that selfexplanations ofien are much simpler if une employs "sloppy" physics inferences, such as blurring the distinction between instantaneous velocity and velocity over a period of time, or between weight as a scalar and weight as a vector. These kinds of sloppy inferences can become difficult habits to break. Indecd, the conceptual blurrings just mentioned are a common source of errors among physics students. It may take careful design in order to find eximple secpuences that block the development of such bad habits. A pseudo-student can help with this design problem.

\section{Cunclusiuns}

Two pseudo-students have been brietly described here. Space does not permit discussion of a third onc, which is being developed in the domain of electronics by David Kieras (personal communication). From a practicial perspective, pseudo-students have both near-term and long-term potential. In the near-term, they help us understand how students learn by proving detailed computational models of the learning processes. This often brings new pedagogical insights even though the models themselves do not find direct use in the education community. Felicity conditions for skill acquisition (VanLehn, 1990a; VanLehn, 1987) are an example of such an insight. In the long term, pseudo-students can probably play an important role in developing high quality instruction. Even though not all instruction needs to be high quality, because its life cycle might be very short or it might be used by only a few student, there are still many applications that demand high quality instruction. Pseudostudents could be a useful tool for providing it.

\section{Acknowledgements}

This research was supported by the Office of Naval Research's Cognitive Sciences Program, contract N00014-88-K-0098, and by the Office of Naval Research's Computer Sciences Division, contract N00014-86-K-0678.

\section{References}

Brown, J. S. \& Burton, R. B. (1978). Diagnostic models for procedural bugs in basic mathematical skills. Cognitive Science, 2, 155-192.

Brown, J. S. \& VanLehn, K. (1980). Repair Theory: A generative theory of bugs in procedural skills. Cognitive Science, 4, 379-426.

Bundy, A., Byrd, L., Luger, G., Mellish, C. \& Palmer, M. (1979). Solving mechanics problenss using meta-level inference. In Proceedings of the 6th International Joint Conference on Arrificial Intelligence. Los Altos, CA: Morgan-Kaufmann.

Burton, R. B. (1982). Diagnosing bugs in a simple procedural skill. In D. H. Sleeman \& J. S. Brown (Eds.), Intelligent Tutoring Systems. New York: Academic. 157-183.

Chi, M.T.H., Bassok, M., Lewis, M., Reimann, P. \& Glaser, R. (1989). Self explanations: How students study and use examples in learning to solve problems. Cognitive Science, 13, 145-182.

Ferguson-Hessler, M.G.M. \& de Jong, T. (1990). Studying physics tests: Differences in study processes between good and poor performers. Cognition and Instruction, 7(1), study proc

Larkin, J. H. (1986). Understanding, problem representation, and skill in physics. In S. F. Chipman, J. W. Segal \& R. Glaser (Ed.), Thinking and learning. Hillsdale, NJ: Erlbaum.

Pirolli, P. \& Bielaczyc, K. (1989). Enpirical analyses of self-explanation and ransfer in learning 10 program. In G. Ohlson \& E. Smith (Ed.), Proceedings of the Anmual Conference of the Cognitive Science Society. Hillsdale, NJ: Erlbaum.

Resnick, L. (1982). Syntax and semantics in learning to subtract. In T. Carpeter, J. Moser \& T. Romberg (Ed.), Addition and subtraction: A developmental perspective. Hillsdale, NJ: Erlbaum.

Resnick, L. B. \& Omanson. S. F. (1987). Learning to understand arithmetic. In R. Glaser (Ed.), Advances in Listructional Psychology. Hillsdale, NJ: Erlbaum. 
Schank, R.C. (1986). Explanation Patterns: Understanding mechanically and creatively. Ifillsdale, NJ: Erlbaum.

van Harmelea, F. \& Bundy, A. (1988). Explanation-based generalisation = partial evaluation. Artificial latelligence, 36, 401.412.

Vanlechn, K. (1982). Bugs are not enough: Empirical studies of bugs, impasses and repairs in procedural skills. The Journal of Mathe'matical Behavior, 3(2), 3-71.

VanLehn, K. (1986). Arithmetic procedures are induced from examples. In J. Hiebert (Ed.), Conceptual and Procedural Knowled de: The Case of Mathematics. Hillsdale, NJ: Erlbaum.

VanLehn, K. (1990). Alind Bugs: The origins of procedural misconceptions. Cambridge, MA: MIT Pless.

VanLehn, K. (1990). Explanation-based learning of correctness: Towards a model of the selfexplanation effect. Submitted to the 1990 Conference of the Cognitive Science Society.

VanLehn, K., Brown, J. S., \& Greeno, J. G. (1984). Competitive argumentation in conpusational theorics of cognition. In W. Kinsch, J. Miller \& P. Polson (Ed.), Merhods and Tacrics in Cognitive Science. Hillsdale, NJ: Erlbaum. 